\title{
Análisis cariotípico de Capsicum pubescens R\&P (Solanaceae) "rocoto"
}

\author{
Karyotype analysis of Capsicum pubescens R\&P (Solanaceae) "rocoto"
}

Misael Guevara, María Siles y Olga Bracamonte*

\section{RESUMEN}

Análisis cariotipico de Capsicum pubescens R\&P (Solanaceae). Los cromosomas han sido descritos, comparados y dibujados, usando una técnica de coloración modificada, C. pubescens tiene un número cromosómico diploide $2 n=24$, de los cuales 11 pares son metacéntricos y 1 par submetacéntrico.

Palabras clave: cromosomas, orceina, bandas, Capsicum, rocoto

\section{ABSTRACT}

Karyotype analysis of Capsicum pubescens (Solanaceae). Chromosomes are described, compared and illustrated, using a modified orcein staining lechnique. C. pubescens has a diploid number of $2 n=24$ chromosomes, 11 pairs are metacentric and one pair is submetacentric.

Key words: Chromosomes. Orcein-bands, Capsicum, rocoto.

\section{INTRODUCCIÓN}

Los ajíes, pertenecientes al género Capsicum, son oriundos de las regiones tropicales del Nuevo Mundo. Las especies domesticadas reconocidas actualmente son $C$. annum (Syn. C. frutescens L.), C. baccatum L., C. pubescens R\&P y C. sinense J. (Brako \& Zarucchi, 1985).

Se trata de un cultivo prometedor debido a sus características fenológicas tales como corto periodo de desarrollo, contenido en minerales (Cárdenas M., 1969), vitaminas A y C; además de capsicina, sustancia a la que se atribuyen propiedades farmacológicas (NRC, 1989).

Se han realizado estudios de germoplasma considerando los aspectos morfológicos, fisiológicos y taxonómicos. En cuanto al estudio citogenético, se sabe que las especies domes- ticadas y algunas silvestres del género Capsicum son diploides $2 n=22$; sin embargo, es la primera vez que se hace el cariotipo de $C$. pubescens.

Para la descripción y análisis de los cromosomas, es ampliamente conocido que en mamíferos, la coloración con Giemsa ha dado buenos resultados y permitido la identificación cromosómica en numerosas especies animales.

En vegetales, la tinción con orceína acética ha permitido obtener numcrosa información (Darlington \& La Cour, 1969). Así mismo cabe indicar que la mayor cantidad de datos proviene del análisis de metafases somáticas.

En 1964, Levan y col. proponen la clasificación de los cromosomas basada en la posición de la constricción primaria donde se encuentra localizado el centrómero, lo que origina dos brazos de longitud variable. 
La relación de los brazos se expresa me. diante índices de proporcionalidad que comparan algunos parámetros (longitud del brazo largo, longitud del brazo corto). Con estos datos se obtiene una relación que permite clasificarlos como mediales (m), submediales ( $\mathrm{sm}$ ), subterminales (st) y terminales ( $t$ ).

La constricción secundaria y los satélites son características de algunos cromosomas; los primeros unen segmentos de un mismo brazo del cromosoma en línea recta, los últimos corresponden a las regiones teloméricas que se presentan como abultamientos a continuación de una constricción secundaria. Las constricciones secundarias son regiones cromosómicas que como las primarias presentan una tinción tenue o negativa. Ambas estructuras son características de algunos cromosomas como el caso de Vicia faba (Shing V. P. \& T. Lelley, 1982), Amarylidaceas (Naranjo,1985) o en humanos 13, 14, 15, 21, 22 (Gonzales Santander, op cit.).

En el presente trabajo se ha realizado el estudio citogenético de Capsicum pubescens R\&P, conocido en el Perú como "rocoto", con la finalidad de que se constituya en una herra mienta de evaluación y caracterización cromosómica.

\section{MATERIAL Y MÉTODOS}

El material biológico correspondió a semillas de C. pubescens R\&P, fruto color rojo, procedente del programa de hortalizas de la Universidad Nacional Agraria La Molina (UNALM), Lima, Perú.

Las preparaciones citológicas para el análisis cromosómico fueron obtenidas a partir de raicillas de $2,3 \mathrm{~cm}$ de longitud, cortadas entre las 10 am y las $12 \mathrm{~m}$ en época de invierno ( $\mathrm{T}^{\circ}$ $\pm 18^{\circ} \mathrm{C}$ ). Fueron pretratadas con 8 -hidroxiquinoleina $0,002 \mathrm{M}$ por 3 horas y después lavadas con agua destilada. Se fijaron en Carnoy
(Etanol-acético, 3:1) durante 20 minutos; fueron transferidas a $\mathrm{HCl} 5 \mathrm{~N}$ durante 10 min para pasar luego a solución Targa; finalmente fueron coloreadas en orceína lacto-acética $2 \%$. La preparación de placas metafásicas se hizo por aplastamiento de las puntas de las raicillas en una gota de colorante frio.

Para el análisis cromosómico, el cariotipo fue organizado tomancio como patrón el tamaño de los cromosomas. Tentativamente los homólogos fueron pareados y colocados en orden decrecicnte de tamaño. La caracterización morfológica de los cromosomas se hizo de acuerdo a lo propuesto por Levan (1964), y la nomenclatura fue tomada de forma similar a los cromosomas de maíz (Aguiar-Perecin y col., 1985).

\section{RESULTADOS}

Se analizaron 200 placas metafásicas, en las que se encontraron células con 22 cromosomas; igualmente se encontraron algunas con 24 cromosomas, 25 y 26 ; sin embargo al realizar el análisis porcentual se encontró que un $97 \%$ de las placas analizadas tenían como número cromosómico 22. Los datos oblenidos han permitido elaborar una tabla de frecuencias de los números cromosómicos de las diferentes muestras (Tabla 1) a partir de las cuales se pudo establecer que el número diploide de cromosomas de C'. pubescens es $2 \mathrm{n}=24$.

El estudio citogenético se inició desde el periodo de interfase, lo que permitió la identificación del nucleolo. Hacia la prometafase la cromatina se encuentra bastante condensada aunque no lo suficiente como para evidenciar cromosomas definidos; sin embargo es en este momento de la vida celular en que se observan las ligaciones que son segmentos intercromatínicos (Guevara M., 1975; Salas, R., 1991); ésias persisten hasta la metafase 
Tabla 1. Frecuencia de los números cromosómicos en Capsicum pubescensR\&P. Conteo realizado en 10 placas metafásicas seleccionadas al azar.

\begin{tabular}{crr}
\hline & \multicolumn{2}{c}{ Frecuencia de placas metafísicas } \\
\cline { 2 - 3 } $\begin{array}{c}\text { Número de cromosomas } \\
\text { por metafase }\end{array}$ & Cantidad & $\%$ \\
\cline { 2 - 3 } & 2 & 1,0 \\
22 & 194 & 97,0 \\
25 & 1 & 0,5 \\
26 & 3 & 1,5 \\
\hline & & 100,0 \\
\hline
\end{tabular}

manteniendo unidos a los cromosomas, lo cual ha dificultado en forma considerable su dispersión.

Las metafases convencionales nos muestran cromosomas consistentes, debidamente elongados, con la aparición en muchos casos de lo que suele llamarse handas espontáneas. La presencia de estas bandas, que se tiñen con la orceína lacto-acética, ayuda considerablemente en la identificación de los homólogos, siendo que se muestran relativamente constantes.

Luego de establecido el número cromosómico se analizaron las metafases con el objeto de elaborar el cariotipo; para ello se toman las longitudes medias de los cromosomas, esto porque se han notado variaciones inclusive entre los homólogos. Se tomaron como medidas las longitudes de los brazos largo y corto del cromosoma y eso ha permitido clasificar a los cromosomas en dos tipos, mediales y submediales. Posteriormente esto ha servido de referencia para la elaboración del cariolipo y idiograma correspondiente (Fig. 1, 2).
Los 11 primeros pares son inediales $(\mathrm{m})$ y el último par es subterminal (st), según la clasificación de Levan et al. (1964). Los 11 pares cromosómicos han sido denominados usando números arábigos descle el par 1 al 12 . Los pares 1 y 2 prescitan en el brazo corto una región intensamente coloreada con la orceína que sigue a una constricción secundaria; estos cromosomas probablemente serían los cromosomas nucleolares. Cabe resaltar que estos dos pares siempre presentan la región intensamente coloreadi en el brazo corto, lo que los diferencia del resto del complemento, pero la constricción secundaria no siempre se observa claramente. EI cuarto par presenta dos bandas delgadas en el brazo corto (p), una es telomérica y la otra pericenrromérica, y una banda telomérica en el brazo largo (q), que permite diferenciarlo del tercer par.

El análisis de los diferentes parámetros cromosómicos ha permitido la total identificación de los cromosomas de C. pubescens, así como la elaboración del cariotipo correspondiente. Los cromosomas ordenados, muestran que los II primeros pares cromosómicos son de posición centromérica medial (m) y de 


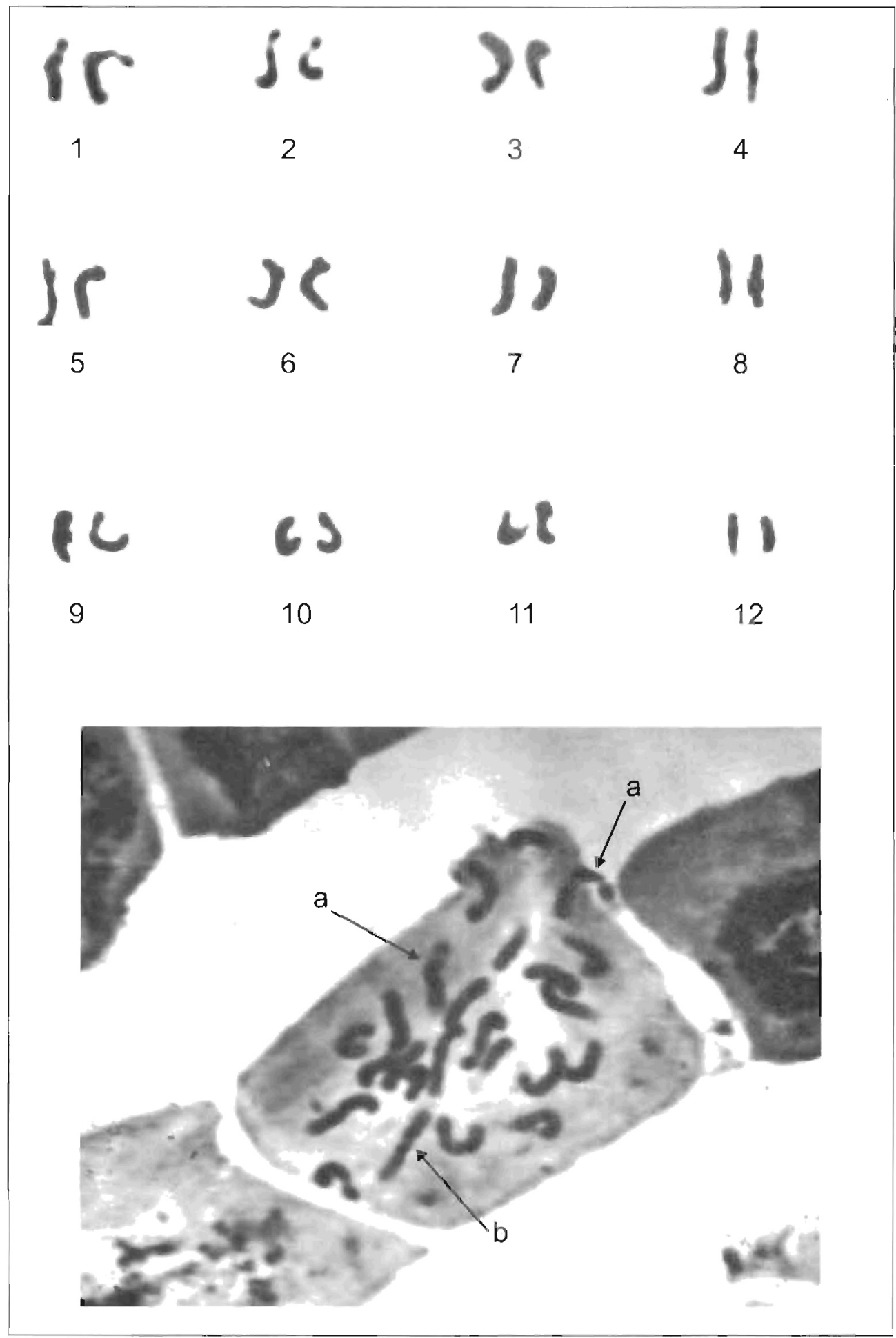

Fig. 1 Cariotipo de Capsicum pubescens R\&P con la placa metafásica correspondiente (600x). En ella se muestran (a) constricciones secundarias (b) constricciones primarias. 
Tabla 2. Clasificación de los pares cromosómicos de Capsicum pubescens R\&P. Los valores presentados son el promedio tomado de diez cariotipos seleccionados al azar.

\begin{tabular}{rccccc}
\hline Pares & $\mathrm{BC} / \mathrm{C}(\mathrm{i})$ & $\% \mathrm{BC}$ & $\% \mathrm{BL}$ & $\% \mathrm{C}$ & $\begin{array}{c}\text { Tipo } \\
\text { cromos. }\end{array}$ \\
\hline 1 & 0,5 & 4,6 & 5,4 & 10,1 & $\mathrm{~m}$ \\
2 & 0,5 & 4,6 & 5,4 & 10,1 & $\mathrm{~m}$ \\
3 & 0,4 & 3,9 & 5,4 & 9,3 & $\mathrm{~m}$ \\
4 & 0,4 & 3,9 & 5,4 & 9,3 & $\mathrm{~m}$ \\
5 & 0,4 & 3,9 & 5,4 & 9,3 & $\mathrm{~m}$ \\
6 & 0,4 & 3,9 & 4,6 & 8,6 & $\mathrm{~m}$ \\
7 & 0,4 & 3,9 & 4,6 & 8,6 & $\mathrm{~m}$ \\
8 & 0,4 & 3,1 & 4,6 & 7,8 & $\mathrm{~m}$ \\
9 & 0,4 & 3,1 & 3,9 & 7,0 & $\mathrm{~m}$ \\
10 & 0,4 & 3,1 & 3,9 & 7,0 & $\mathrm{~m}$ \\
11 & 0,4 & 3,1 & 3,9 & 7,0 & $\mathrm{~m}$ \\
12 & 0,2 & 1,6 & 3,9 & 5,5 & $\mathrm{st}$ \\
\hline
\end{tabular}

* m: medial; st: subterminal, según la nomenclatura propuesta por Levan et al. ( 1964 ), citado por Spororno (1985)

morfología metacéntrica y el último par subterminal (st), en relación al índice centromérico y de morfología acrocéntrica, como se puede apreciar en la tabla 2.

\section{DISCUSIÓN}

La coloración convencional se realizó con orceina lacto-acética: la aparición de las llamadas bandas espontáneas en un gran número de melafases permitió la identificación de los cromosomas homólogos. La presencia de estas bandas estaría relacionada con la utilización de la solución fijadora, el HCl y la posterior coloración con orceína. En vegetales, el bandeo cromosómico no es tan efectivo como en animales debido a la presencia de la pared celular y de características propias de los tejidos vegetales (Chattopadhyay y Sharma, 1988).

Una buena fijación es esencial para clarificar el citoplasma y obtener una buena coloración. El $\mathrm{HCl}$ es considerado el principal agente para resolver bandas cromosómicas (Chattopadhyay y Sharma, 1988). En el presente estudio, la hidrólisis con $\mathrm{HCl} 5 \mathrm{~N}$ a temperatura ambiente, utilizada primariamente para clarificar el citoplasma vegetal, estaría preparando el material para la obtención de estas bandas intercalares cuando posteriormente se colorea con la orceína lactoacético.

En el rocoto, los dos primeros pares cromosómicos presentan una banda terminal en el brazo corto, claramente diferenciada; esta porción va seguida por una estrangulación (o vacío) corta, débilmente teñida, que evidenciaria una constricción secundaria. La porción terminal intensamente teñida es punto de localización del satélite o ricgión "satt".

Tanto la consuricción secundaria como el satélite varían en tamaño en las clistintas placas metafásicas, lo cual se duhería al grado de condensación de los cromosomas, que iam- 


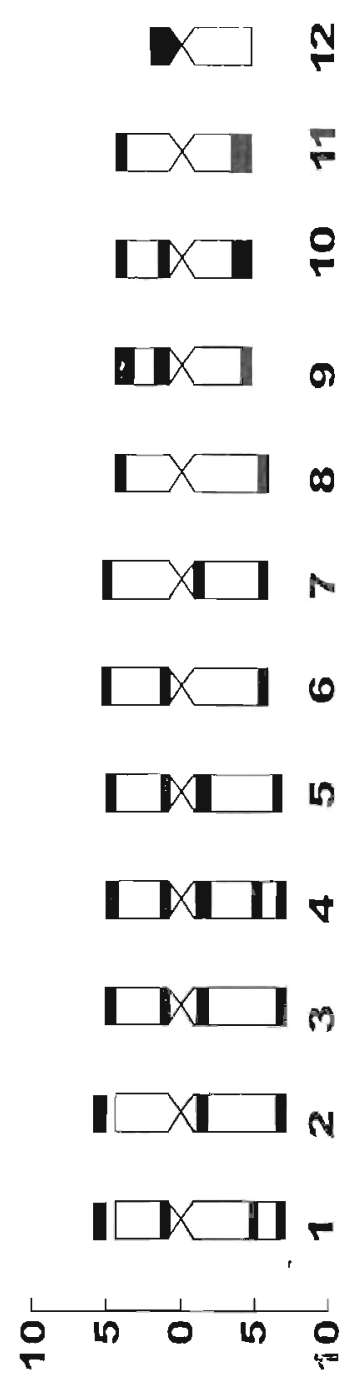

Fig. 2 Cariograma de Capsicum pubescens R\&P muestra la posición de ias bandas heterocromáticas, así como los satelites en los cromosomas 1 y 2.

bién varía de muestra en muestra. Significa que su presencia es constante en todas las metafases, variando sólo su longitud; esto es característico de cromosomas vegetales, como puede comprobarse en el caso de Vicia faba (Swanson 1968, Singh y Lelley 1982), en Amarilidaceae (Naranjo y col., 1982) Gonzales Santander (1976), señala que en vegetales se presenta mayor variabilidad con respecto a constricciones secundarias y satélites; esto lo estaríamos observando en el presente estudio.
En C. pubescens, "rocoto", se observa en algunos casos falta de resolución en algunas bandas intercalares debido al grado de espirilización del cromosoma, lo que depende del estado celular y del uso del antimitótico. Es sabido que los cromosomas vegetales no revelan bandas $\mathrm{G}, \mathrm{R}$ y $\mathrm{Q}$. debido precisamente a su gran espirilización (Grcilbuber, 1977).

En C. pubescens "rocoto", las bardas que observamos con la tinción con orceína, l lamadas también "bandass espontáneas", podrían 
estar relacionadas con el patrón de bandas C; esto último necesita comprobarse.

Hemos utilizado la hidroxiquinoleína como antimitótico. Se sabe que esta sustancia inactiva el huso, no ocasiona ningún obstáculo para la separación cromosómica durante el aplastado, y los brazos cromosómicos se contraen equitativamente. Las constricciones primarias se vuelven conspicuas, de modo que los centrómeros pueden analizarse fácilmente. Es ampliamente utilizada en vegetales y es especialmente adecuada para especies con cromosomas grandes (Sharma y Sharma, 1972). En el rocoto, la hidroxiquinoleína es especialmente adecuada porque la contracción que alcanzan los brazos permite identificar el centrómero sin ninguna dificultad en la mayoría de los casos lo que resulta una ventaja, sobre todo teniendo en cuenta que el bandeo C no revela heterocromatina centromérica en la especie.

El método de bandeo " $C$ " revela heterocromatina constitutiva cuya identidad química es el DNA altamente repetitivo y el agrupamiento de las secuencias repetidas. Esta característica no se pierde cuando la heterocromatina constitutiva se descondensa; por ello se le puede identifical en los núcleos interfásicos, revelándose a la tinción Giemsa después cle un proceso de denaturaciónrenaturación en los cúmulos de heterocromatina intensamente teñidos. En C. pubescens, se observan los núcleos interfásicos con cúmulos heterocromatínicos más o menos gruesos, generalmente asociados a la membrana nuclear; esto último nos estaría sugiriendo la presencia de heterocromatina telomérica, lo que se confirma al analizar los núcleos metafásicos, visualizándose los cromosomas mitóricos con bandas muy leñidas en los telómeros; si bien es cierto que la heterocromatina constitutiva se localiza preferent:mente en la regiones centroméricas de los cromosomas - de ahi el nombre de bandas "C".-, actualmente ya no puedo considerarse una excepción cuando se la localiza en las regiones teloméricas de los cromosomas. De hecho, se presenta así no sólo en especies con cromosomas pequeños como Phaseolus iunatus (Bracamonte, 1992) o Cyclanthera pedata (Gonzales, 1998), tanto como en especies que presentan cromosomas de tamaño mediano como Allium cepa (Curso Iberoamericano de Biología Celular, 1986) e incluso en cromosomas grandes como los de Vicia faba (Singh \& Lelley, 1982).

Los núcleos parcialmente digeriúos y teñidos con cromocentros intensamentc coloreados indican que la técnica de bandeo " $\mathrm{C}$ ", modificada para la especie, es positiva. $C$. pubescens presenta cromosomas de tamaño mediano con bandas heterocromatinicas en las regiones teloméricas de los brazos, lo que nos indica que la especie es pobre en heterocromatina constitutiva. Aún no se puede presentar un patrón de Bandas $C$ debido principalmente a que no se han podido obtener metafases con cromosomas adecuadamente individualizados.

\section{CONCLUSIONES Y RECOMENDA- CIONES}

1 El númern cromosómico es de $2 \mathrm{n}=24$, para Capsicum pubesecens R\&P

2 El cariolipo presenta 12 pares de cromosomas, 11 de los cuales son mediales (m) y el último par subterminal (st).

3 Los dos primeros pares presentan constricción secundaria.

4 Se recomienda continuar con el estudio cromosómico para identificar los cromosomas nucleolares.

\section{LITERATURA CITADA}

Aguiar-Perecin. D. E.; M.R.L. y C. G. Vosa 1985. "C" Banding in Maize II Identification of somatic chromo-somes. Heredity, 54: 37-42. 
Brako L. y J. L. Zarucchi. 1993. Catalogue of the flowering Plants and Gymnosperms of Perú. Missouri Botanical Garden, $1286 \mathrm{pp}$.

Bracamonte G., O. 1992. Citogenética en Phaseolus lunatus L. Tesis para optar el Grado de Magister Scientiae. UNALM. Lima-Perú.

Cárdenas M. 1969. Manual de Plantas Económicas de Bolivia, II plantas condimenticias, Familia Solanaceae, Capsicum spp., 2." edición.

Comings, D. E. 1978. Mechanisms of chromosome banding and implications for chromosome structure. Ann Rew Genet 12:25-43.

Chattopadhyay y Sharma. 1988. Tratamiento ácido para bandeo con orceína, Stain Technology, Vol .63, N. ${ }^{\circ}$ 5, 1988.

Darlington, C. D. y La Cour. 1969. The Handling of Chromosomes.

Dille, J. E. 1988. A technique for C-banding plant chromosomes with wright stain. Stain technology 63(5):271275.

Gonzales, R. A. 1998. Estudio Citogenético de Cyclanthera pedata Schard "cai-hua". Tesis UNMSM.

Greilhuber, J. 1977. Why Plant Chromoso-mes do not show G-Bands? Theor Appl Genet 50:121-124.

Guevara, P. M. 1975. CromocentroCentromero E parametro Ectópico Em Cromossomos politecnicos de varios tecidos de Rhynchosciara angelae Nonal e Pavan (1951).
Ciencia e Cultura, vol. 27(10): 11311140. Brasil.

Gonzales Santander, R. 1976. Introduccion a la citogenetica Humana, Impreso por Selecciones Gráficas España, 285 pp.

Levan, A; Fredge y A. Sandberg 1964. Nomenclature for centromeric position in chromosomes. Hereditas, 52: 201-220.

Naranjo, C. 1985. Carioptipo de Place azaae. Boletín Sociedad Argentina de Botánica, 24: 197-199.

Salas, A. R. 1991 . Ligaciones y su posible Rol en los cromosomas politénicos de Telmatoscopus albipunctatus WiIlinsto, 1983 (Diplora; Psy-chodidae). Informe para optar el Título Profesional de Biólogo con mención en Genética. IINMSM. Lima, Perú.

Sharma, A. K. and A. Sharma. 1972. Chromosome Techniques (Theory and Practice).

Singh, V. P. and Lelley T. 1982. Giemsa CBanding Karyotipe of Vicia narbonensis as compared to Vicia fabct. Fabia Newsletter No.4, 22-23.

Spotorno, A. E. 1985. Conceptos y Métodos en Cariología Descriptiva y Comparada. De: El Núcleo, los cromosomas y la evolución. Editado por R. Fernández-Donoso. UNESCO. 1985

Vosa, C.G. 1989. Chromosome banding: plants. Genome Vol. 31, N. ${ }^{\circ} 1474-$ 475. 